\title{
Prioritization Function for Packet Data Scheduling in OFDMA Systems
}

\author{
I. Gutiérrez \\ Enginyeria i Arquitectura La Salle, \\ Ramon Llull University, \\ Ps. Bonanova, 8. \\ 08022 - Barcelona (Spain). \\ Phone: +34 932902490 \\ igutierrez@salle.url.edu
}

\author{
F. Bader \\ Centre Tecnològic de \\ Telecomunicacions de Catalunya \\ (CTTC), Avda. Canal Olímpic, \\ 08860-Castelldefels (Spain) \\ Phone: +34 936452911 \\ faouzi.bader@cttc.es
}

\author{
J.L. Pijoan \\ Enginyeria i Arquitectura La Salle, \\ Ramon Llull University, \\ Ps. Bonanova, 8. \\ 08022 - Barcelona (Spain). \\ Phone: +34 932902490 \\ joanp@salle.url.edu
}

\begin{abstract}
In this paper, the authors developed a new prioritization function integrated within a new resource allocation process for an OFDMA system. In this paper a new prioritization function is proposed where the resource allocation problem is divided in two phases; the minimum requirements satisfaction, and; the spectral efficiency maximization of the unallocated resources. Compared with the Proportional Fair (PFS) and the Prioritization Function (PRF) algorithms whose have been designed based on the average bit rate requirements without considering neither the states of the buffers or the VBR nature of the traffic, the proposed scheme overcome these restrictions by the support of a designed Packet Data Scheduler (PDS) based on the input buffers status.
\end{abstract}

\section{Categories and Subject Descriptors}

C.2.1 [Network Architecture and Design]: Packet-switching networks, Wireless communication

\section{C.2.5 [Local and Wide-Area Networks]: Access schemes}

\section{General Terms}

Algorithms, Performance.

\section{Keywords}

OFDMA, Scheduling, Resource Allocation, Cross Layer, Quality of Service (QoS).

\section{INTRODUCTION}

To achieve a successful broadband wireless access solution, the IEEE 802.16 subcommittee has released a series of standards for WiMAX (worldwide interoperability for microwave access). From a technical viewpoint, WiMAX is a feasible alternative to the wired internet access solutions such as cable modem and DSL. Nevertheless, from the commercial viewpoint, whether the promise of WiMAX will be materialized still depends on its revenue rate to telecom operators and its service quality to the subscribers. As one of the most expected features, WiMAX is supposed to have the

Permission to make digital or hard copies of all or part of this work for personal or classroom use is granted without fee provided that copies are not made or distributed for profit or commercial advantage and that copies bear this notice and the full citation on the first page. To copy otherwise, or republish, to post on servers or to redistribute to lists, requires prior specific permission and/or a fee.

Wireless Internet Conference'08,Nov. 17-19, 2008, Maui, Hawai, USA.

Copyright 2008 ICST 978-963-9799-36-3. \$5.00. capability of accommodating a variety of traffics [1][2], including data transfer, voice, and video. Correspondingly, four types of service, that is, Unsolicited Grant Service (UGS), Real-Time Polling Service (rtPS), Non- Real-Time Polling Service (nrtPS), and Best Effort (BE), are defined by WiMAX Forum. Among them, UGS, rtPS, and nrtPS are classified into the category of QoS guaranteed services. To handle a multiservice WiMAX access network of heterogeneous traffic load, the resource management scheme that can efficiently allocate radio and bandwidth resources to different subscribers and services is essential.

Most of the existing scheduling functions represent a trade off between spectral efficiency (obtained by adapting the transmission to the channel state) and fairness (imposed from the upper layers in order to balance each user throughput). Scheduling policies based on Weighted Fair Queuing techniques have been designed for maximizing both the system throughput and fairness among users [3]. One scheduling policies used in the $3 \mathrm{G}$ networks is the Proportional Fair Scheduling (PFS) [4]-[6], but it considers full buffers and only Constant Bit Rate (CBR) streams. However in real world multimedia networks have to deal with different traffic types, often Variable Bit Rate (VBR) streams, and furthermore very strict packet delay requirements must be fulfilled. Recent trends in packet scheduling consider cross-layer strategies such those proposed in [7]-[9], where the resource allocation and the data scheduling are performed together. Liu et al. proposed in [7] a scheduling where a priority is assigned to each user according to its instantaneous channel and service status. In [7], the channel state is obtained directly from the average received signal to noise ratio (SNR), whereas the service status is obtained from the longest packet waiting time, i.e. the head of line (HOL) delay. The same principle is extended in [8] to the WiMAX Orthogonal Frequency Division Multiple Access (OFDMA) system, where the priorities are also assigned as a function of the subchannel. Furthermore, in [9] Soo et al. proposed to prioritize the packets according to a named "emergency factor" which is the ratio between the HOL and the maximum delay constraint, thus users with higher emergency factor are scheduled first.

The authors propose a new data prioritization function where scheduling and resource allocation are carried out jointly. The whole scheduling and resource allocation process are divided into two steps; the minimum requirements allocation, and; the spectral efficiency maximization. In the first step each user gets a number of allocated resources

\footnotetext{
${ }^{1}$ This work was partially supported by the European ICT-2008-211887 PHYDYAS project, and the Spanish National project Project contract/code: TEC2006-1 0459/TCM.
} 
depending on the queued packets, and the delay associated to each packet. In the second step, in case when there are enough available resources are assigned to the users with better channel state information in order to increase the spectral efficiency. The performance of the proposed function is evaluated and compared with other scheduling functions from similar researches.

The rest of the paper is organized as follows. In Section II, the system model considered is introduced. The proposed scheduling function is then detailed in Section III, and the corresponding simulation results are analyzed in Section IV. Finally, conclusions are stated in section V, where the benefits and drawbacks of the proposed approach are summarized.

\section{SYSTEM DESCRIPTION}

The downlink transmission mode in a IEEE 802.16 PMP (Point to Multi-Point) system is considered in this proposal, with one single cell and a total of $K$ mobile stations (MSs) within the cell area, a perfectly synchronization between the base station and the mobile stations, and a free interference environment. Since we deal with channel reciprocity between uplink and downlink the Time Division Duplexing (TDD) is considered. As a result, no Channel Quality Indicators (CQI) needs to be fed back in the uplink mode.

The communication frame is formed by $N_{s}$ OFDM symbols with a frame duration $T_{\text {frame }}$ seconds. The total bandwidth $B W$ is formed by $N_{c}$ subcarriers where only $N_{\text {used }}$ are active, the remaining are used as guard tones. The active sub-carriers include both the pilot subcarriers and the data subcarriers which will be mapped and distributed over different subchannels according to the specific subcarrier permutation scheme. The minimum allocation unit referred as Basic Radio Unit (BRU), is then obtained as one subchannel by one Time Transmission Interval (TTI), which may vary between 1 and 3 OFDM symbols depending on the permutation scheme. Adjacent subcarrier permutation (also known as Band AMC) is assumed, where the subcarriers assigned to each subchannel are adjacent in the spectrum. In this case, as it is shown in [10], the multiuser diversity is increased due to large differences between the channel state within each BRU measured over the whole set of users.

Following the flow of the data from upper layers to lower layers, first the data for each user is classified according its class of service and mapped to a service flow. To simplify the analysis and without loss of generality, during the paper we consider that each MS is associated to a unique service flow. The packets generated from each service flow are then buffered independently (i.e. there is one buffer per user) and each incoming packet is time stamped. New packets are received periodically at the input buffers at a rate that depends on the class of service. Four services classes are defined in the IEEE 802.16, the Unsolicited Grant Service (UGS), the Real-Time Polling Service (rtPS), the Non-real Time Polling Service (nrtPS) and the Best Effort (BE) service [10]. The maximum packet delay, the average bit rate and the peak bit rate, as well as the packet rate must be specified for each individual application.

The scheduler then transmits the packets according to the specific prioritization algorithm and the number of resources allocated by the radio resource allocation block. The combination of both tasks (scheduling and allocation) is detailed in hereunder in Section III. Once both processes are completed, the physical layer codifies and modulates the data prior to transmitting it through the radio channel.

On the other hand, there is also a flow of control information from the physical layer to the medium access layer, who is reported with the maximum number of bits that can be transmitted to each user in each BRU according to the Modulation and Coding Schemes (MCS) available.

\section{III.RESOURCE ALLOCATION AND SCHEDULING SCHEME}

One of the main objectives of the resource allocation and scheduling mechanisms is to guarantee the different service flow QoS constraints, and maximize the spectral efficiency. Each service flow is mapped into one class of service with fixed QoS constraints during the whole communication time. These constraints are mainly: the average bit rate, the peak bit rate and the minimum bit rate, the maximum tolerated delay per packet, and the average bit/packet error rate.

In order to select which resources will be assigned to each service flow (and thus to each MS), each $i$-th service flow has a priority value assigned during each frame over each $n$-th subchannel (the channel is assumed constant during the whole communication frame). The priority values are obtained according to two factors: the channel quality index (CQI), and the amount of required resources for each requested service. In [7]-[9], the priority is computed according to the product of the CQI and a delay satisfaction indicator. However, the algorithms have been designed based on average bit rate requirements without considering neither the states of the buffers or the VBR nature of the traffic.

To overcome these restrictions, the authors propose a Packet Data Scheduler (PDS) based on the input buffers status (i.e. the size of each $p$-th packet, $L_{i, p}$, and the delay of each packet, $\left.\tau_{i, p}\right)$, and the channel metrics proposed in [7]. Then for each $i$-th service flow we define a scheduling priority value $\varphi_{i}(n)$ over each $n$-th subchannel as

$$
\begin{aligned}
& \boldsymbol{\varphi}_{i}(n)=\left\{\begin{array}{l}
\min \left(\frac{b_{i}}{b_{\max }}, 1\right) \cdot \frac{\eta_{i}(n)}{\eta_{\max }}, \text { if } \forall p^{\prime} \rightarrow \tau_{i, p^{\prime}}<\left(\tau_{\max , i}-\Delta \tau\right)(1) \\
P_{\text {urgency }} \frac{\eta_{i}(n)}{\eta_{\max }}, \text { otherwise }
\end{array}\right. \\
& b_{i}=\left\{\begin{array}{l}
T_{\text {frame }} \sum_{p=1}^{P} \frac{L_{i, p}}{\tau_{\text {max }, i}-\Delta \tau-\tau_{i, p}}, \text { if } \forall p^{\prime} \rightarrow \tau_{i, p^{\prime}}<\left(\tau_{\text {max }, i}-\Delta \tau\right)(2) \\
\sum_{p^{\prime}} L_{i, p^{\prime}}, \text { otherwise }
\end{array}\right.
\end{aligned}
$$

where $\min (x, y)$ takes the minimum value of $x$ and $y \cdot \eta_{i}(n)$ is the spectral efficiency achieved by the highest modulation and coding schemes that can be used within the $n$-th subchannel giving a BER lower than a predefined upper bound value. $\eta_{\max }$ is the spectral efficiency achieved by the highest MCS. When $\eta_{i}(n)=0$, it means that the $n$-th subchannel of the $i$-th MS experiments a deep fading. In this case the priority becomes zero. $b_{i}$ is the minimum number of bits that should be transmitted in the actual frame in order to achieve a delay for each $p$-th packet lower than $\left(\tau_{\max , i}-\Delta \tau\right)$, where $\tau_{\max , i}$ is the maximum allowed delay for the class of service associated to the $i$-th service flow, and $\Delta \tau$ represent a guard time (i.e. $\left.\Delta \tau=0.2 \tau_{\max , i}\right) . \quad b_{\max }$ is a normalization factor which is the 
maximum number of bits that can be transmitted within a frame using the highest MCS. Note that in case any packet from the $i$-th service flow exceeds its maximum delay, the term $b_{i} / b_{\max }$ is substituted by an urgency factor (i.e. $P_{\text {urgency }}=10$ ), which boosts the priority given to the $i$-th service flow in order to avoid packet drops due to excessive packet delay. From(2), it is also observed that in case where a buffer is empty, the priority given to that service flow is zero.

The allocation process then assigns iteratively the BRUs to those users with highest priority at each iteration. Once a user has its minimum requirements allocated $b_{i}$, the user is extracted from the poll of demanding users. In case a service flow is not allocated the minimum resources (since all the resources are exhausted or due to its channel conditions), its priority in the next frame will be automatically increased according to (2) in order to flush those bits that should have been transmitted in previous frames. Afterwards when all the users' requirements are fulfilled and in case there are still BRUs to allocate, the priorities are updated to

$$
\boldsymbol{\varphi}_{i}(n)=\left\{\begin{array}{ll}
\frac{\eta_{i}(n)}{\eta_{\max }}, & \text { if } b_{i}>0 \\
0, & \text { otherwise }
\end{array},\right.
$$

with

$$
b_{i}=\sum_{p} L_{i, p}
$$

Thus, during this second phase the user with higher carrier to noise ratio is scheduled in each BRU, maximizing the spectral efficiency.

When all the BRU have been allocated, or the input buffers have been emptied, the end of the joint resource allocation and packet scheduling process is achieved. Since the packets must be received in the correct order, the data from the buffers is extracted from older packets to the newer ones (as in a First In First Out queue). The packet delay is then measured as the difference between the times elapsed since the packet is queued at the buffer and the time where all its bits have been transmitted.

\section{IV.SIMULATION RESULTS}

Table I summarise the system parameters. During all the analysis $K$ users are dropped at different positions having a uniform distribution within the cell area (the maximum cell radius is fixed to $1 \mathrm{Km}$ ). The position of the MS remains fixed during the whole simulation time, while the speed of the MSs is only used to determine the Doppler effects, and the channel coherence time [12]. A simulation time of 50 seconds (10.000 frames) has been chosen to ensure the convergence of the user's service flows and the performance metrics. The full process is repeated with the MSs dropped at new random locations. The number of drops simulated for each scenario is 25 , which makes the results independent of the BS to MS distances. As already mentioned, each user has only one assigned service flow. Five classes of services are considered during the simulations whose main parameters are summarized in Table II, and the traffic models according to parameters in [11]. The channel estimation is assumed ideal at the Base Station, and packet retransmission not considered. Furthermore, we considered that the target Bite Error Rate (BER) for all the service classes is $\mathrm{BER}<10^{-6}$. In this case, the minimum Effective SNR per MCS with the mandatory punctured convolutional coding defined in the IEEE 802.16 standard [10] (constraint length 7 and native code rate 1/2) are: [7, 8.7, 9.6, 11.2] for QPSK, [13.9, 15.6, 16.6, 18] for 16QAM, and $[20,21.7,22.7,24.3]$ for 64QAM with coding rates of $1 / 2$, $2 / 3,3 / 4$ and $5 / 6$ respectively. To obtain the Effective SNR (which is the SNR required to give the same BER considering an AWGN channel), the channel values inside each subchannel are merged using the geometric mean [12].

The obtained performance of the proposed prioritization function is then evaluated and compared with the PFS [5], and the Prioritization Function (PRF) in [8]. In case of PFS, Eq. (1) is substituted by,

$$
\boldsymbol{\varphi}_{i}(n)=\frac{1}{T h_{a}(t)} \cdot \frac{\eta_{i}(n)}{\eta_{\max }}
$$

where $T h_{a}(t)$ is the average throughput obtained by a moving average window with $\alpha$ as the window length and $T h_{i}(t)$ the instantaneous throughput, thus

$$
T h_{a}(t)=\frac{1}{\alpha} T h_{i}(t)+\left(1-\frac{1}{\alpha}\right) T h_{a}(t-1), \quad \text { with } T h_{i}(t) \geq 0 .
$$

On the other hand, according to the PRF in [8], the scheduling priority is given by,

$$
\boldsymbol{\varphi}_{i}(n)= \begin{cases}\beta_{\operatorname{CoS}} \cdot \frac{\eta_{i}(n)}{\eta_{\max }} \frac{1}{F_{i}}, & \text { if } F_{i} \geq 1 \\ \beta_{\operatorname{CoS}}, & \text { otherwise }\end{cases}
$$

where $\beta \in(0,1]$ is the priority associated to the class of service of the $i$-th service flow (i.e. $\beta=1$ for $U G S, \beta=0.8$ for $r t P S$, $\beta=0.6$ for $n r t P S$ or $\beta=0.4$ otherwise). $F_{i}$ is the service satisfaction level associated to the first packet on the buffer queue (the head of line) which is obtained by

$$
F_{i}=\frac{\tau_{\max , i}-\tau_{\max , P}}{\Delta \tau} .
$$

Regarding to the resource allocation process used in PFS and PRF only the first phase is considered fixing the number of bits per frame that can be transmitted equal to the number of buffered bits (i.e. $b_{i}=L_{i}(t)$ ). For the PFS case the latency scale is fixed equal to 10 frames (i.e. $\alpha=10$ ).

First the performance of the mentioned schedulers is compared in case of rtPS traffic. In this case each user stream is modelled as a VBR, with an average bit rate of $380 \mathrm{Kbps}$. For the $r t P S$ traffic, when a packet is not transmitted within the maximum delay $\tau_{\max }$, then the packet is deleted from the queue and discarded. Then two parameters have been studied: the packet delay statistics and the packet loss rate, i.e. the number of discarded packets divided by the total number of packets (packets discarded plus packets transm itted). Figure 1 shows the cumulative density function of the packet delay for 50 and 100 users. First, we focus on the $K=50$ case where, as it is shown in Fig.1, all the schemes achieve a delay lower than the maximum allowed $(50 \mathrm{~ms})$, in fact the 99 th percentile (delays such that $p(x<$ delay $)>0.99)$ is found at $25 \mathrm{~ms}, 25 \mathrm{~ms}$ and $35 \mathrm{~ms}$ for the PDS, the PFS and the PRF respectively. Furthermore, the packet loss rate for each scheme is 0 for the PDS, 1.6e-5 for the PFS and $2.22 \mathrm{e}-3 \cdot$ for the PRF. Next, for the $K=100$ case we can observe that the PFS is the one that gives lower packet delays, and that the PDS sends most of the packets when the urgency factor is applied. During the simulations the guard

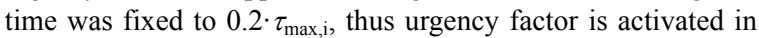


this case when $\tau_{i, p}>\left(\tau_{\max , \mathrm{i}}-\Delta \tau\right)=0.4 \mathrm{~ms}$. Now, for $K=100$ the packet loss rate for each scheduling function is $0.0824,0.1375$ and 0.2505 for the PDS, the PFS and the PRF respectively. Again for the PDS scheduler, although most of the packets are sent when they are nearly to expire, a lower packet loss rate is achieved. Joining both results (packet delay and packet loss rate) it is shown that for real time applications the PDS scheduler is able to improve the performance of the system by guaranteeing the QoS requirements.

In Fig. 2 the same analysis is performed in case of non-real time (nrt) traffic. The main difference from the previous case is that packets belonging to the $n r t$ traffic are not discarded in case its delay exceeds the maximum value. Then we can observe that the PFS provides around $95.9 \%$ of the packets with a delay lower than $0.3 \mathrm{~s}$, whereas the PRF achieves only a $87.6 \%$ percent of the packets in time. However, we can appreciate that the PDS achieves a 99.61. In other words, the 99 percentile for the packet delay is $260 \mathrm{~ms}, 12.75 \mathrm{~s}, 1.465 \mathrm{~s}$. The difference in performance is dramatic for the nrt traffic since only the PDS achieves a delay lower than the maximum allowed for the $99 \%$ of the packets. Actually, for the PDS scheduler we can appreciate that at $0.24 \mathrm{~s}$ the packets are speed up due to the urgency factor. Moreover, the cumulative density function of the spectral efficiency measured in each frame is also shown in Fig. 3 ( $n r t$ traffic is applied). For the PRF and the PFS we can observe a big step at $5 \mathrm{bits} / \mathrm{s} / \mathrm{Hz}$. This means that users with good channel conditions obtain a large priority and thus, the scheduler give access to the channel to the users with best channel conditions. On the other hand, the PDS gives a larger range of spectral efficiencies. We can attribute this to the fact that with the PDS all the users try to obtain every frame their minimum resources in order to get a delay lower than the maximum value. Furthermore, we also observe that despite the mentioned large percentage of frames with maximum spectral efficiency, the PRF and the PFS lead to higher number of frames than the PDS with low spectral efficiency. Thus the PFS and the PRF moves between high and low spectrally efficient frames, whereas the PDS produces lower variations on the spectral efficiency.

Then, the performance of the PDS in case of mixed traffic is shown in Fig.4. In this scenario a total amount of $K=50$ users are simulated, where 10 users require $n r t P S, 13$ users require $r t P S, 10$ users are browsing internet files (World Wide Web service), 5 are downloading files according with the File Transfer Protocol (FTP), and finally 12 users require UGS connections for applications such as Voice over IP. The total measured downlink throughput is $26.5 \mathrm{Mbps}$. The maximum delay for each service is indicated in Table II, while the delay for the WWW and FTP services has been assumed as $\tau_{\max }=60 \mathrm{~s}$ and $\tau_{\max }=90$ s respectively. It is clearly shown in Fig. 4 that each traffic type achieves a maximum packet delay lower than its maximum value. Actually, the 99th percentile for the delay sensitive applications is found at $100 \mathrm{~ms}, 35 \mathrm{~ms}$ and $20 \mathrm{~ms}$ for the $n r t P S$, the $r t P S$ and the UGS respectively, much lower than its maximum values.

In Fig. 5 the fairness of the proposed scheduling function is evaluated. In this case the simulated traffic is nrtPS type. The number of MSs within the cell is $K=15$. Therefore, taking into account that an average data rate of $2 \mathrm{Mbps}$ is demanded per user, an average system throughput of $30 \mathrm{Mbps}$ is required. The maximum delay per packet is $300 \mathrm{~ms}$, however for the $n r t P S$ the packets are not discarded if they exceed the maximum delay. To evaluate the fairness of the different schedulers, the delay statistics are obtained as a function of the distance between the BS and the MS which is sampled at intervals of $100 \mathrm{~m}$ starting at $50 \mathrm{~m}$. We can observe that the average delay for all the prioritization functions is below the maximum delay. Furthermore, we can also observe that the variation of the average packet delay is lower for the PFS scheduler as it was expected. Another important result that might be observed in Fig.5 is the lower delay obtained for the PDS scheduler for those users closer to the BS. The reason for that comes from the two steps design of the resource allocation process, serving first the minimum requirements and later serving those users with better channel conditions. On the other hand, when studying the 99th percentile obtained at each of the sampled distances another important conclusion is drawn. Despite the 99th percentile experiences a larger variation for the PDS prioritization function than for the PFS, we observe that at further distances the maximum delay is well upper bounded, being able to guarantee a delay lower than $200 \mathrm{~ms}$ for $99 \%$ of the packets. On the other hand, we can observe that the delay measured for the PFS and the PRF schedulers is above its maximum value for the users in the cell border.

Therefore, despite the PDS initially implies an increase in computational complexity since it requires more information about the buffers status (i.e. each packet must be time stamped for the PDS scheduler and an independent buffer per service flow is required), we have shown its superiority for real and non-real time applications. Moreover, for the proposed PDS scheduling function there is no necessity to update the priorities each time a BRU is allocated, thus the computational complexity is then reduced compared to the PFS and the PRF functions. Nevertheless, we have also shown that the PDS can easily manage different traffic types as well as managing fairly the resources despite different average SNR per user.

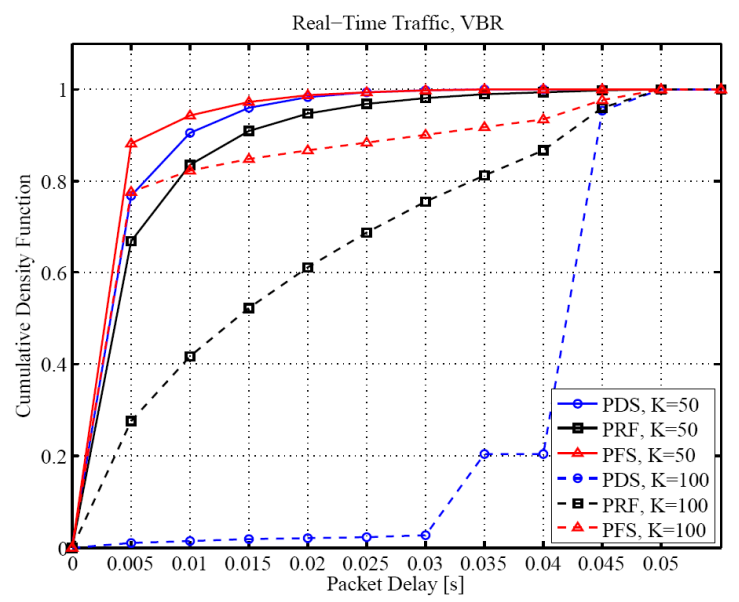

Figure 1. Performance comparison for $r t P S$ traffic, $K=\{50$, $100\}$ users, of the proposed prioritization function against the PFS and the PRF functions. 


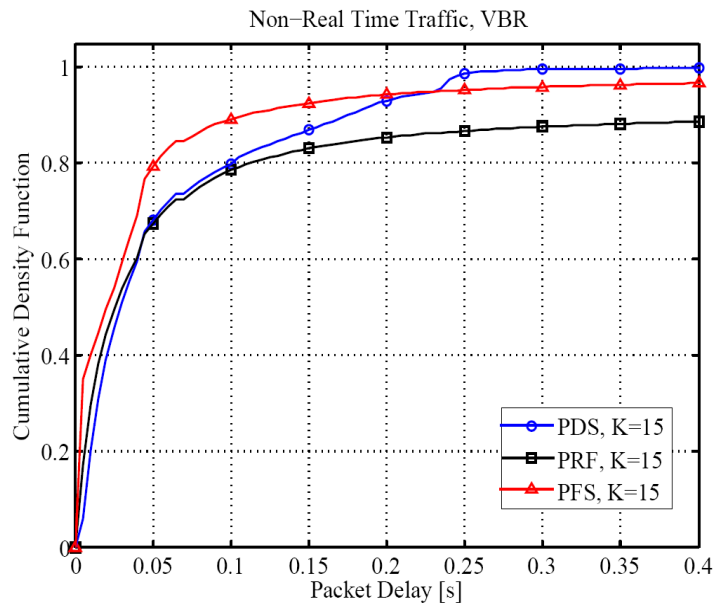

Figure 2. Performance comparison for $\mathrm{n} r \mathrm{PS}$ traffic, $K=15$ users, of the proposed prioritization function against the PFS and the PRF functions.

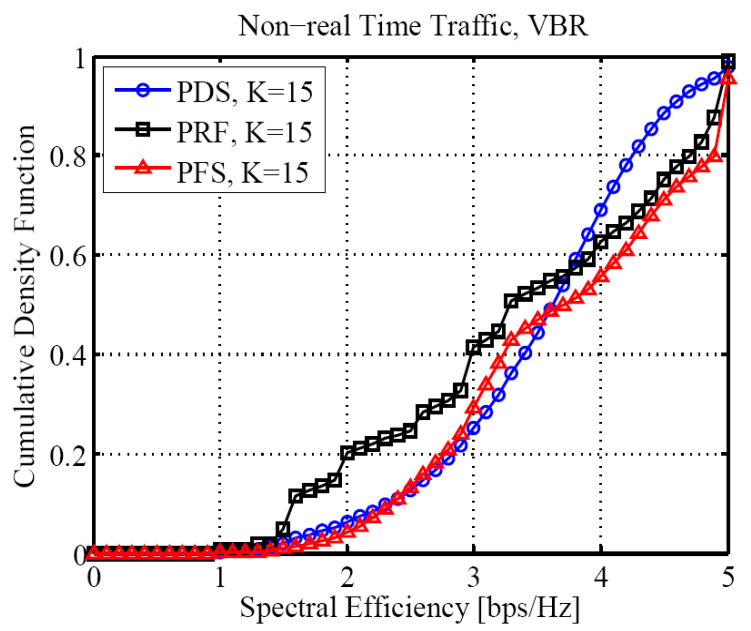

Figure 3. Spectral efficiency statistics in case of $n r t P S$ traffic and $K=15$ users.

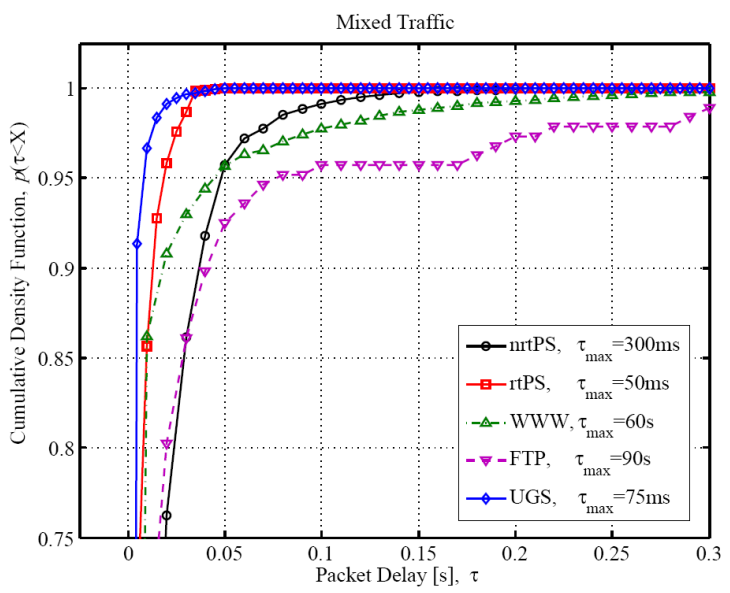

Figure 4. Cumulative density function of the packet delay for mixed traffic from $K=50$ users, and the PDS function.

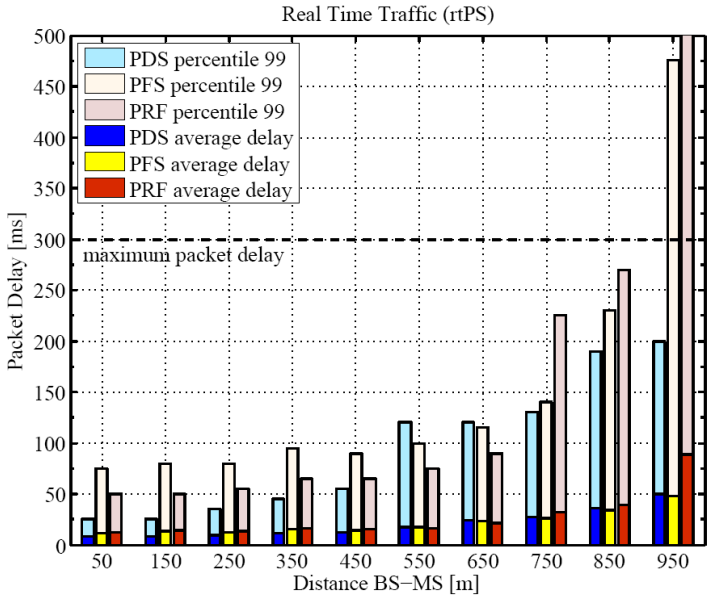

Figure 5. Packet delay statistics as function of the distance between BS-MS for $n r t P S$ traffic and $K=15$ users.

Table I - System parameters.

OFDMA Air Interface and System Level configuration

\begin{tabular}{|l|l|}
\hline Carrier Freq & $3.5 \mathrm{GHz}$ \\
\hline Bandwidth & $20 \mathrm{MHz}$ \\
\hline Sampling Frequency & $22.857 \mathrm{Msps}$ \\
\hline Subcarrier Permutation & Band AMC \\
\hline FFT length & 2048 \\
\hline Cyclic Prefix & $12.5 \%$ \\
\hline \# of used subcarriers & 1728 \\
\hline \# of subcarriers per subchan. & 18 \\
\hline \# of OFDM symbols per BRU & 3 \\
\hline \# of data symbols per BRU & $48($ efficiency $=8 / 9)$ \\
\hline Modulation & $\{4,16,64\}$-QAM \\
\hline Channel coding & Punctured Convolutional \\
\hline Channel Model & Pedestrian B \\
\hline Channel estimation and CQI & Ideal \\
\hline Shadowing standard deviation & $5 \mathrm{~dB}$ \\
\hline BS Tx power & $49 \mathrm{dBm}$ \\
\hline Thermal noise & $-174 \mathrm{dBm}$ \\
\hline BS antenna gain and pattern & $14 \mathrm{~dB}$, Sectorial \\
\hline MS antenna gain and pattern & $0 \mathrm{~dB}$, Omnidirectional \\
\hline Path loss, urban environment & $139.57+$ \\
& $28 * 10 g_{10}(\mathrm{R}[$ Km] $)$ \\
\hline Other Link budget parameters & $\begin{array}{l}\text { BS height }=30 \mathrm{~m}, \\
\text { MS heigh }=1.5 \mathrm{~m}, \\
\text { MS Noise Figure }=5 \mathrm{~dB}, \\
\text { Connectors Loss }=2 \mathrm{~dB} \\
\text { Cell Radius }=1000 \mathrm{~m}\end{array}$ \\
\hline Frame duration, $T_{\text {frame }}$ & $5 \mathrm{~ms}$ \\
\hline OFDM symbols in the DL & 30 \\
\hline
\end{tabular}

Table II. Simulated classes of service parameters.

\begin{tabular}{|c|c|c|c|c|}
\hline $\begin{array}{c}\text { Class of } \\
\text { Service }\end{array}$ & $\begin{array}{c}\text { Average } \\
\text { bit rate }\end{array}$ & $\begin{array}{c}\text { Peak } \\
\text { bit rate }\end{array}$ & $\begin{array}{c}\text { Max. } \\
\text { Delay }\end{array}$ & $\begin{array}{c}\text { Packet } \\
\text { Rate }\end{array}$ \\
\hline$r t P S$ & $380 \mathrm{Kbps}$ & $2 \mathrm{Mbps}$ & $50 \mathrm{~ms}$ & $10 \mathrm{pps}$ \\
\hline$n r t P S$ & $2 \mathrm{Mbps}$ & $10 \mathrm{Mbps}$ & $300 \mathrm{~ms}$ & $10 \mathrm{pps}$ \\
\hline$w w w$ & N/A & $2 \mathrm{Mbps}$ & N/A & Variant \\
\hline$f t p$ & N/A & $10 \mathrm{Mbps}$ & N/A & Variant \\
\hline$U G S$ & $15 \mathrm{Kbps}$ & $15 \mathrm{Kbps}$ & $50 \mathrm{~ms}$ & $20 \mathrm{pps}$ \\
\hline
\end{tabular}




\section{CONCLUSION}

In this paper, the authors developed a prioritization function for packet data scheduling integrated within a new resource allocation process for an OFDMA system. The achieved performance has shown that with proposed algorithm it possible to guarantee the maximum delay for delay sensitive applications (i.e $r t P S$ and $n r t P S$ ), while at the same time a high spectral efficiency is obtained by exploiting the multiuser diversity of those unallocated resources. Furthermore, in comparison with the PFS and the PRF, the proposed algorithm outperforms them in almost the analyzed scenarios at the expense of a slight increase in computational cost. The PDS can easily manage different traffic types as well as managing fairly the resources despite different average SNR per user.

\section{REFERENCES}

[1] ITU-T Recommendation Y.2001 (12/2004) - General overview of NGN, Dec., 2004.

[2] Recommendation ITU-R M.1645, "Framework and overall objectives of the future development of IMT-2000 and systems beyond IMT-2000". June, 2003.

[3] V. Bharghavan, L. Songwu, T. Nandagopal, "Fair queuing in wireless networks: issues and approaches," IEEE Personal Communications, vol.6, no.1, pp.44-53, Feb 1999

[4] H.J. Kushner, P.A. Whiting, "Convergence of proportional-fair sharing algorithms under general conditions," IEEE Transactions on Wireless Communications, vol.3, n.4, July 2004.

[5] H. Kim,Y. Han, "A proportional fair scheduling for multicarrier transmission systems". IEEE Communications Letters, March 2005, Vol. 9, Iss. 3, pp. 210- 212

[6] B. Classon, et al., "Overview of UMTS Air-Interface Evolution", IEEE $64^{\text {th }}$ Vehicular Technology Conference, 2006. Fall. 2006.

[7] Q. Liu, X. Wang, G. B. Giannakis, "A Cross-Layer Scheduling Algorithm With QoS Support in Wireless Networks", IEEE Transactions on Vehicular Technology, Vol. 55, No. 3, May 2006.

[8] L. Wan, W. Ma, Z. Guo, "A Cross-Layer Packet Scheduling and Subchannel Allocation Scheme in 802.16e OFDMA System", Proc. on Wireless Communication and Networking Conference, 2007.

[9] S. Soo, D. Geun, W. Sook, "Cross-layer Design of Packet Scheduling and Resource Allocation in OFDMA Wireless Multimedia Networks", Proc. on IEEE $63^{\text {rd }}$ Vehicular Technology Conference, May, 2006, Melbourne.

[10] IEEE 802.16e-2005, IEEE standard for local and metropolitan area networks, Part 16: Air interface for fixed and mobile broadband wireless access systems, amendment 2, February 2006.

[11] IST-2001-32620-MATRICE, D1.3, "Specification of the performance evaluation methodology ant the target performance", Dec. 2002.

[12] IEEE 802.16m-08/004r1, "IEEE 802.16m Evaluation Methodology Document", March, 2008. 